\title{
Vision Assisted Laser Scanner Navigation for Autonomous Robots
}

\author{
Andersen, Jens Christian; Andersen, Nils Axel; Ravn, Ole
}

Published in:

Proceedings of the 10th International Symposium on Experimental Robotics 2006 (ISER '06)

Publication date:

2008

Link back to DTU Orbit

Citation (APA):

Andersen, J. C., Andersen, N. A., \& Ravn, O. (2008). Vision Assisted Laser Scanner Navigation for Autonomous Robots. In Proceedings of the 10th International Symposium on Experimental Robotics 2006 (ISER '06) (Vol. 39, pp. 111-120). Springer.

\section{General rights}

Copyright and moral rights for the publications made accessible in the public portal are retained by the authors and/or other copyright owners and it is a condition of accessing publications that users recognise and abide by the legal requirements associated with these rights.

- Users may download and print one copy of any publication from the public portal for the purpose of private study or research.

- You may not further distribute the material or use it for any profit-making activity or commercial gain

- You may freely distribute the URL identifying the publication in the public portal

If you believe that this document breaches copyright please contact us providing details, and we will remove access to the work immediately and investigate your claim 


\title{
Vision Assisted Laser Scanner Navigation for Autonomous Robots
}

\author{
Jens Christian Andersen, Nils A. Andersen, Ole Ravn \\ Automation Ørsted•DTU, Technical University of Denmark, \\ DTU-build. 326, DK-2800 Kgs. Lyngby, Denmark. \\ jca@oersted.dtu.dk naa@oersted.dtu.dk or@oersted.dtu.dk
}

Summary. This paper describes a navigation method based on road detection using both a laser scanner and a vision sensor. The method is to classify the surface in front of the robot into traversable segments (road) and obstacles using the laser scanner, this classifies the area just in front of the robot $(2.5 \mathrm{~m})$. The front looking camera is used to classify the road from this distance and forward, taking a seed area from the laser scanner data and from this estimate the outline of the visible part of the road. The method has been tested successfully on gravelled and asphalt roads in a national park environment.

\section{Introduction}

Autonomous navigation by mobile robots in unstructured or semi-structured outdoor environments presents a considerable challenge. Adequately solving this challenge would allow robotic applications like daily maintenance and inspection of public infrastructure, assisting disabled people, guiding guests, and a large number of other tasks. To achieve the level of autonomy required for such operation, a robot must be able to perceive and interpret the environment, and to use perceived reality in the navigation planning. For autonomous robots to be popular, the production cost should be low, and the ability to cope with the real world should be high. In many urban as well as rural areas the Global Positioning System (GPS) has insufficient coverage to guide the robot along a desired path. Additionally the robot needs a local navigation ability to avoid obstacles. The motivation is therefore to provide the robot with a navigation capability that copes with the real world challenges. The robot should behave reasonably civilized, i.e. stay on the roads, on one side of broader roads, avoid and show intension to avoid obstacles as early as possible, and in general behave as could be expected from a pedestrian.

It is imperative for successful autonomous navigation that the robot is able to identify obstacles (or boundaries) which should not be crossed or collided with. The sensors used for this task are predominantly vision and laser scanners, both combined solutions and each sensor type individually. 
Extraction of relevant features in the environment from the sensor data is an essential foundation for safe navigation. Edge detection in vision systems is one of the possibilities to identify road borders, and had some success already in 1986, as described in [6]. An algorithm that distinguished compressible grass (which is traversable) from obstacles such as rocks using spatial coherence techniques with an omni-directional single line laser was developed by [5]. A detection and tracking method for the vertical edges of the curbstones bordering the road, using a 2D laser scanner, was developed by [8]. Stereo vision has large potential in the related work of [7] and [1]. The DARPA Grand Challenge event in 2004 has spawned a multitude of work related to autonomous navigation and obstacle avoidance, e.g. [3] and [9], and after the 2005 event better results were obtained, and the focus on a combination of laser scanner and vision is maintained.

The approach in this project is to use laser scanner data for classification of the area in front of the robot into traversable and non-traversable segments, and to detect relevant obstacles within the coverage area. Supplement this with a vision sensor capable of finding the outline of the (traversable) road beyond the laser scanner range. The vision approach is to take a seed area - that is classified as traversable by the laser scanner - and use this to encircle the area in the image with similar properties. The detected features - traversable segments, obstacles and road outline - are then fused into a feature map directly used for path decisions.

A national park has been selected as test area (see Fig. 1). The available roads have a surface of either gravel or asphalt. The roadsides are mostly flat with cut grass or rough vegetation. Parts of the route are below large overhanging trees. The traffic is mostly limited to pedestrians, horses and bicycles.

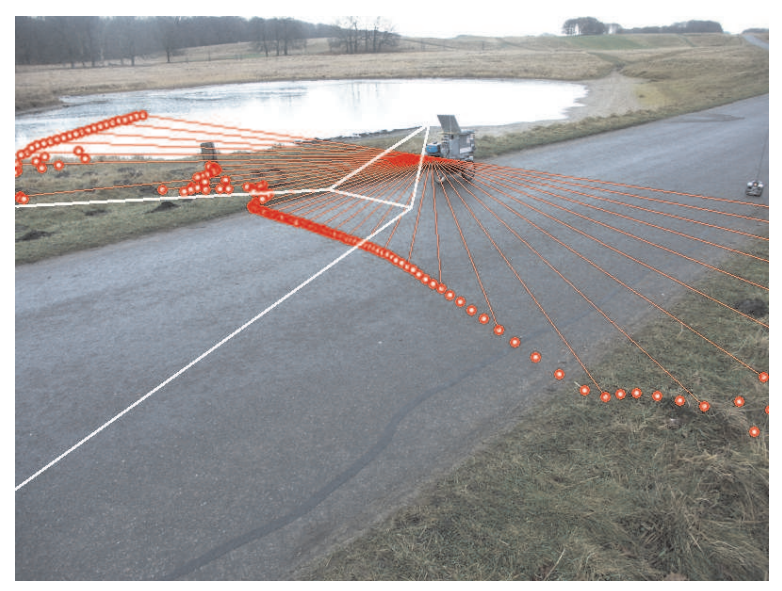

Fig. 1. The robot in the test area with laser scanner measurements and camera coverage 


\section{Vehicle}

The selected vehicle is a robot with about the same weight and terrain capabilities as an electric wheelchair.

The drive system has differential drive and use a combination of odometry and a one-axis gyro to estimate the robot pose $[x, y, \Theta]$.

The robot and the view from the laser scanner and vision are shown in Fig. 1.

The laser scanner is looking down at an angle of $9^{\circ}$ towards the road surface $(\approx 2.5 \mathrm{~m})$ in front of the vehicle. This angle is a compromise between detecting small obstacles (obstacles larger than $5 \mathrm{~cm}$ needs to be avoided), scan rate, obstacle warning distance and ability to cope with terrain curvature. The laser scanner has a $180^{\circ}$ scan width, and the instrumented range is $8 \mathrm{~m}$.

The colour camera has a $71^{\circ}$ horizontal field of view and is oriented towards the road so that the top of the image is just above the horizon.

\section{Terrain classification from laser scanner}

The assumption is that the terrain seen by the laser scanner can be divided into class $\mathcal{C}=\left\{\mathcal{C}_{t}\right.$ (traversable), $\mathcal{C}_{n}$ (not traversable), $\mathcal{C}_{\varnothing}$ (invalid data) $\}$, and that this can be done by a mapping function $\mathcal{M}_{C F}(\mathcal{F}): \mathcal{F} \rightarrow \mathcal{C}$. Here $\mathcal{F}$ is a set of features extracted from single laserscans: $\mathcal{F}=\left\{\mathcal{F}_{h}\right.$ raw height, $\mathcal{F}_{\sigma}$ roughness, $\mathcal{F}_{z}$ step size, $\mathcal{F}_{c}$ curvature, $\mathcal{F}_{t}$ slope, $\mathcal{F}_{w}$ width $\}$.

A roughness value is calculated as deviation from a fitted line for measurements converging a distance of $45 \mathrm{~cm}$ (the wheel base), to emphasize terrain variation with a spatial period shorter than this distance. The roughness feature function $\mathcal{F}_{\sigma}$ divides the measurements into groups based on this roughness value, these groups are then combined and filtered based on the remaining feature functions $\left\{\mathcal{F}_{h}, \mathcal{F}_{z}, \mathcal{F}_{c}, \mathcal{F}_{t}, \mathcal{F}_{w}\right\}$.

Each of these functions increases the probability that the measurements are correctly classified. The method is described in detail in $[4,2]$.

An example of the obtained classification is shown in Fig. 2, where a narrow gravelled road is crossed by a bridle path. The road and the bridle path are first divided into a number of roughness groups as shown in Fig. 2b, these are then filtered down to three traversable segments, one for the road (in the middle) and one each side from the bridle path.

\section{Road outline from vision}

The assumption is that it is possible to estimate the outline of the road by analyzing the image, based on a seed area in the image classified as traversable by the laser scanner. 


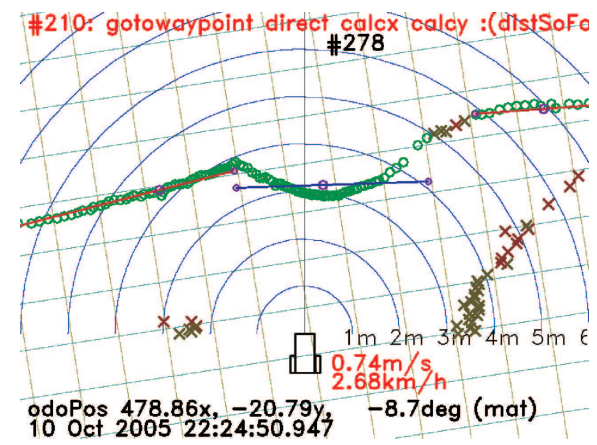

(a)

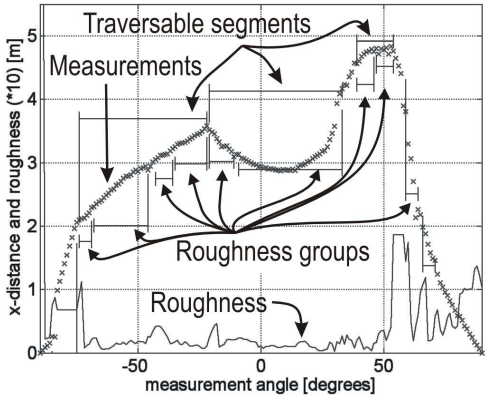

(b)

Fig. 2. Data from a gravelled road crossed by a bridle path. The laser scanner measurements are shown in (a) as circles (traversable) and crosses (not traversable), the rough grass on the road edges before the bridle path is visible left and right of the robot. The road is the area with the high profile (about $15 \mathrm{~cm}$ higher at the center). On both sides are relative flat areas from the bridle path. The segmentation into roughness groups and traversable segments are shown in (b)

The main features describing the road are its homogeneity. But there may be variation in the visual expression due to e.g. shadows, sunlight, specular reflections, surface granularity, flaws, partially wet or dry surface and minor obstacles like leaves.

The road detection is therefore based on two features: the chromaticity $\mathbf{C}$ and the intensity gradient $\nabla I$.

The chromaticity value $\mathbf{c}$ is based on an RGB image as shown in (1)

$$
\mathbf{c}=\left[\begin{array}{c}
c_{\text {red }} \\
c_{\text {green }}
\end{array}\right]=\left[\begin{array}{c}
r /(r+g+b)+g_{\text {red }}(\bar{I}-(r+g+b)) \\
g /(r+g+b)
\end{array}\right],
$$

where $g_{\text {red }}(\bar{I}-(r+g+b))$ is a first order compensation for shadows, and $\bar{I}$ and $g_{\text {red }}$ is the linearization point and the gradient for the compensation.

Each pixel $H_{i, j}$ is classified into class $\mathcal{R}=\{$ road, not road $\}$ based on these features. The $\mathcal{R}_{\text {road }}$ classification is defined as

$$
\mathcal{R}_{\text {road }}\left(H_{i, j}\right)=\left\{\begin{array}{l|l}
H_{i, j} & \begin{array}{l}
P_{c}\left(\mathbf{C}\left(H_{i, j}\right)\right)+ \\
P_{e}\left(\nabla I\left(H_{i, j}\right)\right) \\
>K_{\text {limit }}
\end{array}
\end{array}\right\}
$$

where $P_{c}(\cdot)(3)$ is a probability function the based on the Mahalanobi distance of the chromaticity relative to the seed area. $P_{e}(\cdot)(4)$ is based on the intensity gradient, calculated using a Sobel operator. The Sobel kernel size is selected as appropriate for the position in the image, i.e. the lower in the image the larger the kernel $(3 \times 3$ at top and $5 \times 5$ pixels at the bottom for the used $320 \times 240$ image resolution). 


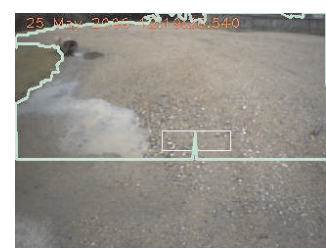

(a)

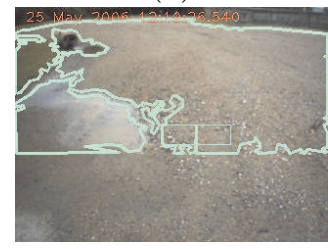

(b)

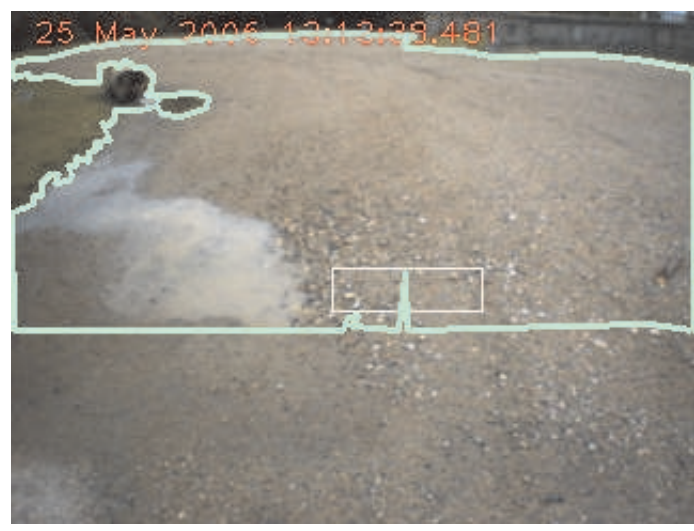

(c)

Fig. 3. Road outline extraction based on chromaticity (a), on gradient detection (b) and combined (c). In the top left corner there is a stone fence, this is not distinguished from the gravel road surface using the chromaticity filter in (a). The gradient filter (b) makes a border to the pit (bottom left). The combined filter (c) outlines the traversable area as desired. The seed area classified by the laser scanner is shown as a (thin) rectangle. The part of the image below the seed area is not analyzed

$$
\begin{aligned}
& P_{c}(i, j)=\left(1+w_{c}\left(\mathbf{c}_{i, j}-\overline{\mathbf{c}}_{w}\right)^{T} \mathbf{Q}^{-1}\left(\mathbf{c}_{i, j}-\overline{\mathbf{c}}_{w}\right)\right)^{-1} \\
& P_{e}(i, j)=\left(1+w_{e}\left[\left|\frac{\partial I(i, j)}{\partial i}\right|^{2}+\left|\frac{\partial I(i, j)}{\partial j}\right|^{2}\right]\right)^{-1}
\end{aligned}
$$

where $\mathbf{Q}$ is the chromaticity covariance for the seed area. The $w_{c}$ and $w_{e}$ are weight factors.

An example of the capabilities of the filter functions is shown in Fig. 3.

The pixels at the road contour are evaluated only, i.e. from the seed area pixels are tested towards the image edge or road border, the road border is then followed back to the seed area.

\section{Obstacle detection}

In each laser scan the measurements outside the traversable segments are divided into spatially separated obstacles if they are close to a traversable segment or closer to the robot.

The obstacle range measurements are merged into convex polygon shapes and merged with obstacles detected in the previous scan.

The obstacle merging assumes that the obstacle may represent a (relatively) flat rough area and allows correlation with obstacles seen at the same position relative to the robot from the previous scan. This allows larger areas of rough vegetation to be represented by one obstacle (within limits). 
A level shift between two traversable segments is further included as an obstacle if the level shift is above the obstacle size limit.

\section{Sensor fusion}

A feature map representation is adapted for sensor fusion and navigation planning. The detected features are the traversable segments from the laser scanner covering ranges up to about $2.5 \mathrm{~m}$ in front of the robot, the vision based road outline from about $2 \mathrm{~m}$ and forward, and the obstacles detected from the laser scanner data.

Each traversable segment $S_{j}^{k}$ extracted by the laser scanner in the most recent scan $k$ is correlated with traversable segments from previous scans $S^{k-i}$, forming a set of traversable corridors $B$ as shown in (5). A correlation exists if the segments overlap by more than a robot width

$$
B_{i}=\left\{S_{a^{0}}^{k}, S_{a^{1}}^{k-1}, S_{a^{2}}^{k-2}, \cdots, S_{a^{N}}^{k-N}\right\}
$$

where $S_{a}^{k-i}$ is the $a$ th traversable segment found in scan $k-i$.

This corridor of traversable segments gets extended beyond the range of the laser scanner using the road outline from the vision sensor. Intersection lines $S^{k+v}$ (perpendicular to the current robot heading) at increasing intervals are used to extend the laser scanner corridors, as shown in (6)

$$
B_{i}=\left\{S_{b^{1}}^{v 1}, S_{b^{2}}^{v 2}, \cdots, S_{b^{M}}^{v M}, S_{a^{0}}^{k}, S_{a^{1}}^{k-1}, S_{a^{2}}^{k-2}, \cdots, S_{a^{N}}^{k-N}\right\},
$$

where $S_{b}^{v n}$ is the $b$ th intersection segment of intersection line $n$ inside the estimated road outline. See examples in Fig. $6 \mathrm{~b}$ and $7 \mathrm{~b}$.

A number of such corridors may exist, e.g. left and right of obstacles, left and right in road forks or as a result of erroneous classification from the laser scanner or form the vision. A navigation route is planned along each of these corridors considering the obstacles, current navigation objectives and the robot dynamics. The best route is qualified using a number of parameters including corridor statistics. If the vision is unable to estimate a usable road outline then the laser scanner data is used only.

\section{Results}

The method is tested primarily on a $3 \mathrm{~km}$ route in a national park. The navigation is guided by a script specifying how to follow the roads and for how long. At the junctions the script guides the robot in an approximate direction until the next road is found. GPS is used sparsely to determine when a road section is about to end.

The road width estimate and the road width stability can be taken as a performance measure of the vision and laser scanner sensors. 


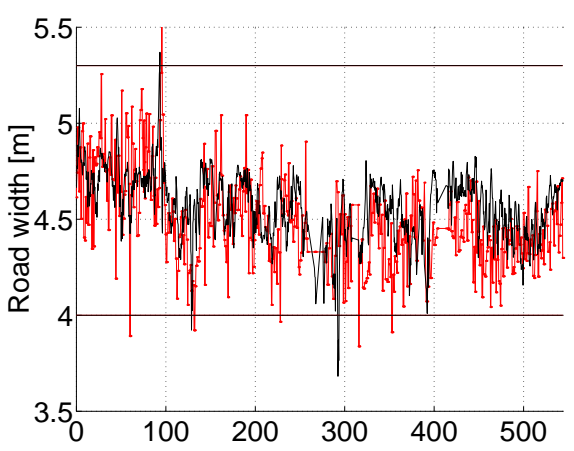

(a) distance $[\mathrm{m}]$

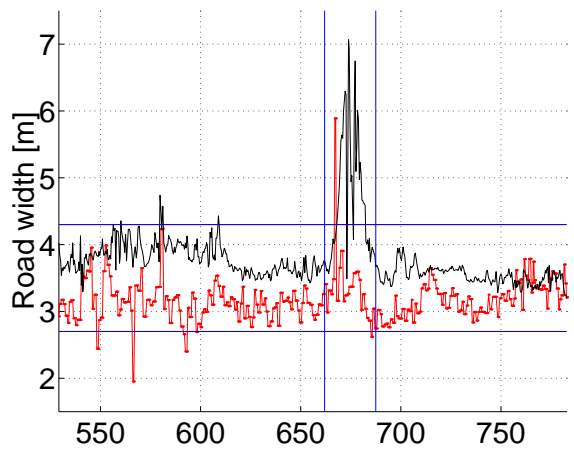

(b) distance $[\mathrm{m}]$

Fig. 4. Road width estimation based on laser scanner (solid black) and vision based (red). A section from a $4.9 \mathrm{~m}$ wide asphalt road (a), and a $3.5 \mathrm{~m}$ wide gravelled road (b) with a side road at about $675 \mathrm{~m}$. The vision ans laser-scanner based road width almost identical on the wide road in (a). The gravelled road (b) is detected with a higher uncertainty, the laser-scanner based estimate is slightly too wide and the vision-based slightly too narrow

Table 1. Road width estimate summary from the data shown in Fig. 4a and 4b is in the first two rows. The last two rows are from a narrow asphalt road in overcast (wet) and sunny weather conditions, respectively. The 'w/n' column is the too wide or too narrow count as a percentage of the measurement count $\mathrm{N}$

\begin{tabular}{lccccccccc}
\hline Road & \multicolumn{1}{c}{ True } & \multicolumn{1}{c}{ Laser based } & \multicolumn{7}{c}{ Vision based } \\
segment & width & mean & $\sigma$ & $\mathrm{N}$ & mean & $\sigma$ & w/n & N & ref \\
\hline Asphalt & 4.9 & 4.7 & 0.17 & 1200 & 4.5 & 0.24 & $1 \%$ & 474 & $4 \mathrm{a}$ \\
Gravelled & 3.5 & 3.7 & 0.48 & 600 & 3.2 & 0.32 & $3 \%$ & 245 & $4 \mathrm{~b}$ \\
Asphalt & $3-4$ & 3.5 & 0.63 & 890 & 2.8 & 0.36 & $2 \%$ & 224 & wet \\
Asphalt & $3-4$ & 3.3 & 0.46 & 482 & 2.8 & 0.53 & $16 \%$ & 79 & sun \\
\hline
\end{tabular}

Figure 4 shows the road width detection from two road sections, a homogeneous asphalt road (a) and a $3.5 \mathrm{~m}$ wide gravelled road (b). The weather conditions were overcast with mild showers. The road width is estimated based on the available data (from (6)) at time of manoeuvre decision.

The vision based road width estimate is in the plane of the robot base, and as the road has a convex profile, the road width in the projected plane is narrower than in reality - the narrow road in Fig. 4b has a higher profile than Fig. 4a.

The road width estimates are summarized in Table 1 for the laser scanner and vision sensor respectively. The laser scanner based estimate shows the correct road width in most cases, with a tendency to include parts of the roadside, especially for the gravelled road where the roughness difference between the road and the roadside is small. The last two rows in Table 1 are 
from a road segment that is partially below large trees - and here the road outline estimates failed in $16 \%$ of the measurements on the day with sunshine, compared to just $2 \%$ in gray and wet weather condition.

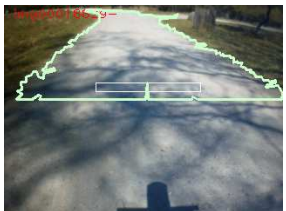

(a)

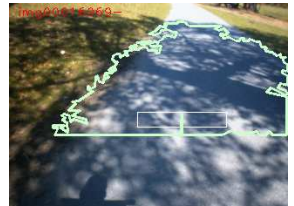

(b)

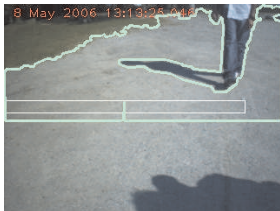

(c)

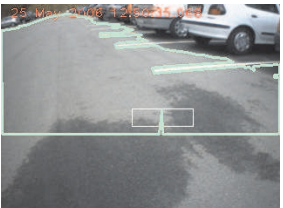

$(\mathbf{d})$

Fig. 5. Shadows and road markings at the limits of the vision sensor capabilities. Unfocused shadows like in (a) and (b) are handled reasonably well. Hard shadows as in (c) and road markings as the white markings in a parking lot (d) are handled as obstacles

The vision based road outline detector does neither cope well with hard shadow lines as shown in Fig. 5c, nor with the painted road markings as in Fig. 5d. Unfocused shadows as in Fig. 5a and Fig. 5b are handled reasonably well. Wet and dry parts of the road are less of a problem for the road outline detector as shown in Fig. 5d.

When the road outline is limited by obstacles as in the situation shown in Fig. 6a, the route possibilities in the feature map (Fig. 6b) will be limited correspondingly, and the result is an obstacle avoidance route initiated at an early stage. The pedestrian can follow the robot intentions as soon as the obstacle avoidance route is initiated, and thus limit potential conflict situations.
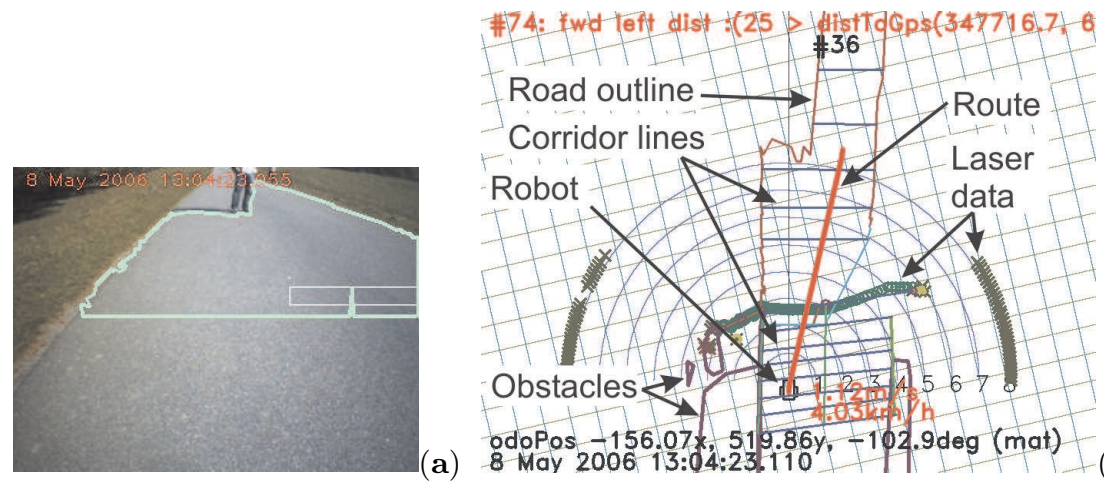

Fig. 6. The pedestrian (and his shadow) is reducing the road outline (a) and an obstacle avoidance route is planned (as shown in (b)) long before the obstacle is seen by the laser scanner 

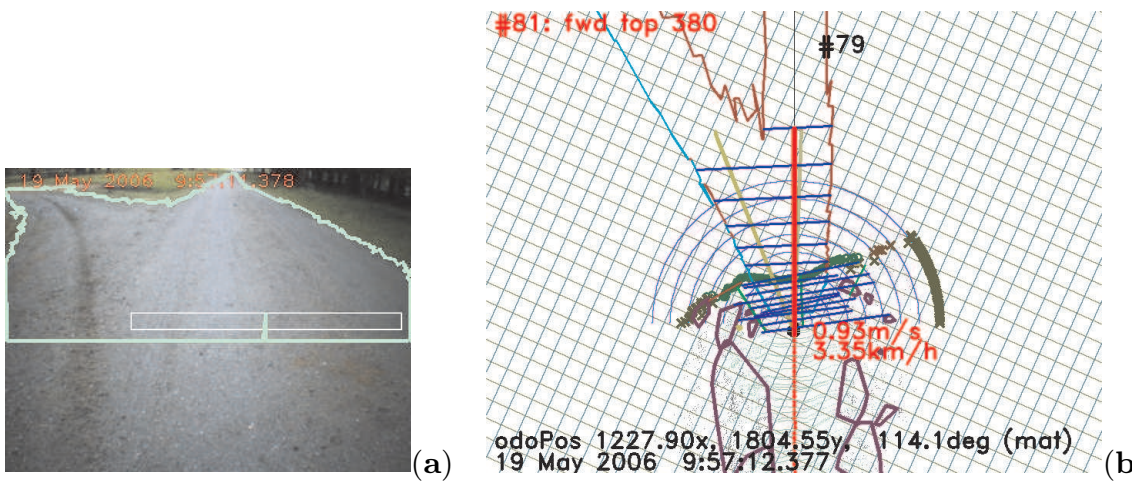

Fig. 7. A road fork is seen by the vision sensor (a) and included in the road outline shown on the feature map (b). The navigation process provides two main alternatives and selects the fork to the right

In junctions and transitions for open areas to a narrower road the vision will often be able to see the exit road or the road alternatives. This can assist the navigation system in planning a route towards the right road at an early stage. In Fig. 7 the road forks and the vision based road outline clearly show the road alternatives in the feature map (Fig. $7 \mathrm{~b}$ ). The route alternatives are shown in the feature map, and the right fork road is selected (as desired).

\section{Conclusion and future work}

This paper has presented a navigation method using a combination of laser scanner and camera to navigate in an outdoor environment on available roads. A test route of about $3 \mathrm{~km}$ is traversed successfully using this navigation method.

At the used robot speeds of $1-1.5 \mathrm{~ms}^{-1}$ navigation is possible using the laser scanner only, but when it is assisted by a vision-based road outline detector the navigation performance increases significantly.

The grass covered roadsides are at times classified as traversable by the laser scanner processing, with vision assistance the robot is able to follow the road edges without entering the grass (in almost all cases).

The robot is able to start obstacle avoidance early and the manoeuvres are thus smoother, and passing of pedestrians gets more relaxed.

In road junctions the vision outline will in most cases be able to provide guidance to available road exits.

The vision road detection is more volatile and fails or has limited performance in a number of situations e.g. focused shadows and road markings. The sensor is furthermore dependant on a reliable seed area from the laser scanner. 
For future work especially two areas are evident, first, the ability to cope with focused and hard shadows should be improved. Secondly, side roads and road alternatives in junctions are not detected in time to allow a seamless transition; improvements here would improve autonomy or reduce the need for planning.

\section{References}

1. J. C. Andersen, N. Andersen, and O. Ravn. Trinocular stereo vision for intelligent robot navigation. In 5th IFAC/EURON Symposium on Intelligent Autonomous Vehicles, Lisboa, Portugal, July 2004.

2. J. C. Andersen, M. R. Blas, N. Andersen, O. Ravn, and M. Blanke. Traversable terrain classification for outdoor autonomous robots using single $2 \mathrm{D}$ laser scans. Integrated Computer-Aided Engineering, 2006.

3. R. Behringer, W. Travis, R. Daily, D. Bevly, W. Kubinger, W. Herzner, and V. Fehlberg. Rascal - an autonomous ground vehicle for desert driving in the darpa grand challenge 2005. In Proceedings of Intelligent Transportation Systems, 2005, pages 644-649. IEEE, 2005.

4. M. R. Blas, S. Riisgaard, O. Ravn, N. Andersen, M. Blanke, and J. C. Andersen. Terrain classification for outdoor autonomous robots using $2 \mathrm{D}$ laser scans. In 2nd int. Conf. on Informatics in Control, Automation and Robotics, ICINCO-2005., pages 347-351, Barcelona, 14-17 September, 2005.

5. J. Macedo, L. Matthies, and R. Manduchi. Ladar-based discrimination of grass from obstacles for autonomous navigation. In Proceedings of Experimental Robotics VII, ISER 2000, Waikiki, Hawaii, pages 111-120. Springer, 2000.

6. R. Wallace, K. Matsuzaki, Y. Goto, J. Crisman, J. Webb, and T. Kanade. Progress in robot road-following. In Proceedings of 1986 IEEE International Conference on Robotics and Automation (Cat. No.86CH2282-2), pages 1615-21 vol 3. IEEE Comput. Soc. Press, 1986.

7. H. Wang, J. Xu, J. I. Guzman, R. A. Jarvis, T. Goh, and C. W. Chan. Real time obstacle detection for agv navigation using multi-baseline stereo source. In Proceedings of Experimental Robotics VII, ISER 2000, Waikiki, Hawaii, pages 561-568. Springer-Verlag, 2000.

8. W. Wijesoma, K. Kodagoda, and A. Balasuriya. Road-boundary detection and tracking using ladar sensing. IEEE Transactions on Robotics and Automation, pages 456-464, 2004.

9. Z. Xiang and U. Ozguner. Environmental perception and multi-sensor data fusion for off-road autonomous vehicles. In Proceedings of Intelligent Transportation Systems, 2005, pages 584-589. IEEE, 2005. 